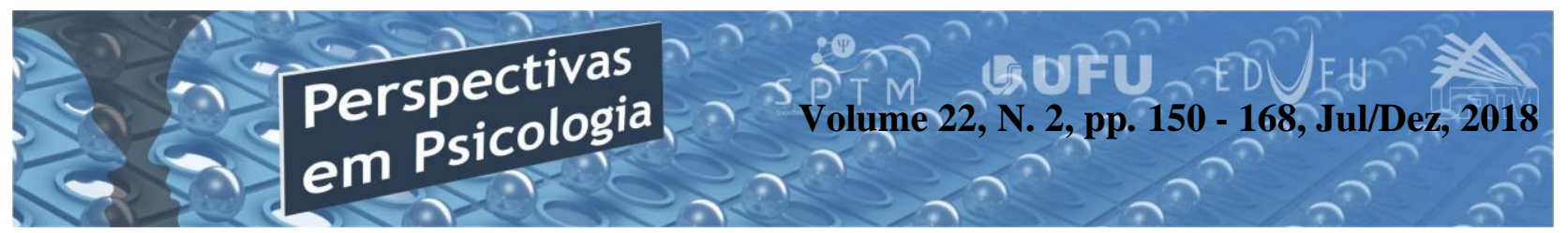

\title{
COMPROVAÇÃO DA ESTRUTURA FATORIAL DA MEDIDA DE CONDUTAS ANTISSOCIAIS E DELITIVAS EM JOVENS
}

\author{
Maria de Fatima de Matos Maia \\ (Universidade Estadual de Montes Claros, Montes Claros - MG) \\ Nilton Soares Formiga \\ (Universidade Potiguar, UNP, Natal - RN) \\ Thatiana Maia Tolentino \\ (Faculdade Santo Agostinho de Sete Lagoas, Sete Lagoas - MG) \\ Celina Aparecida Gonçalves Lima \\ (Universidade Estadual de Montes Claros, Montes Claros - MG) \\ Berenilde Valéria de Oliveira Sousa \\ (Universidade Estadual de Montes Claros, Montes Claros - MG)
}

\begin{abstract}
Resumo
Pesquisadores em distintas áreas das ciências humanas e sociais têm procurado esclarecer os motivos dos jovens apresentarem comportamentos de violência simbólica e intensa. A testagem psicológica responde de forma preditiva aos porquês das condutas transgressoras. Este estudo pretende verificar se a estrutura fatorial da escala de condutas antissociais e delitivas ainda existe. 691 sujeitos de 12 a 19 anos dos dois gêneros participaram do estudo, em distintos níveis do ensino público na cidade de Montes Claros-MG, respondendo a um questionário sociodemográfico e à escala de condutas antissociais e delitivas. Foram utilizados os programas SPSS, para a estatística descritiva, e o AMOS 21.0, para análise fatorial confirmatória. O modelo bifatorial oblíquo revelou melhores indicadores psicométricos, confirmando resultados de estudos no Brasil e fora dele. Assim, as dimensões das condutas antissociais e delitivas vêm se mantendo confiáveis e válidas quanto ao construto psicológico, podendo ser utilizado para mensurar a conduta desviante na população juvenil.
\end{abstract}

Palavras-chave: condutas antissociais; condutas delitivas; jovens estudantes; análise fatorial.

\section{Abstract \\ Evidence of the Factorial Structure of the Measure of Antisocial and Delinquent Conducts in young people.}

Researchers in different areas of the human and social sciences have sought to clarify the motives of young people to exhibit behaviors of symbolic and intense violence. Psychological testing responds in a predictive way to the whys of transgressive behaviors. This study aims to verify if the factorial structure of the scale of antisocial and delusional conducts still exists. 691 subjects from 12 to 19 years of age of both genders participated in the study, at different levels of public education in the city of Montes Claros, Minas Gerais, responding to a sociodemographic questionnaire and to the scale of antisocial and delinquent behaviors. SPSS program was used for descriptive statistics and AMOS 21.0 for confirmatory factor analysis. The two-tailed oblique 


\section{DELITIVAS EM JOVENS}

model revealed better psychometric indicators, confirming results of studies in Brazil and elsewhere. Thus, the dimensions of antisocial and delinquent behaviors have remained reliable and valid regarding the psychological construct, and can be used to measure deviant behavior in the juvenile population.

Keywords: antisocial conducts; delinquent conduct; young students; factor analysis.

\section{Introdução}

A constante preocupação com jovens e suas formas de manifestações diferenciadas e por vezes não aceitas ou incompreendidas remetem a estudos que buscam entender o porquê de determinados comportamentos e/ou atitudes na adolescência. $\mathrm{O}$ envolvimento de adolescentes com condutas desviantes, seja com a formação de gangues, seja através de roubos, uso de drogas, porte de armas e etc. tem sido revelado na mídia em geral e por estudiosos em distintas áreas da ciência humana e social. Isso é considerado um problema não apenas para a sociedade, mas também para a dinâmica das relações interpessoais, condição esta que afeta tanto a saúde pessoal do jovem quanto a da sua família, da escola e das comunidades em que vivem, constituindo-se uma situação de saúde pública (Negreiros, 2008; Jager, Batista, Perrone, Santos, \& Dias, 2014; Formiga, Duarte, Neves, Machado, \& Machado, 2015).

Devido a essa situação complexa, que requer soluções urgentes, ressalta-se a necessidade de pontuar questões que possam esclarecer o motivo de muitos jovens estarem envolvidos em situações que fogem às normas sociais, assim como o seu envolvimento com comportamentos violentos, justamente por serem questões difíceis de repostas instantâneas, seja devido à complexidade do comportamento humano (Vasconcelos, Gouveia, \& Pessoa, 2008), seja pelo isolamento causal em distintas áreas científicas e suas micro e macroteorias sobre esse fenômeno na sociedade contemporânea (Born \& Bradley, 2005; Negreiros, 2008; Silva \& Salles, 2010; Formiga, Duarte e outros, 2015; Morgado \& Vale-Dias, 2014). Diante de um fenômeno tão sério, o qual exige uma intervenção urgente, compreende-se a importância de avaliar esse tipo de conduta com base em aspectos sobre o funcionamento familiar, ocupacional, escolar e social dos adolescentes, mas, também, é necessária uma instrumentalização capaz de avaliar de forma específica e até preditiva esse tipo de comportamento. Com isso, instrumentos de medidas atitudinais e até diagnósticos têm sido propostos, os quais buscam mensurar a frequência, grau e/ou intensidade de conduta desviante no 
desenvolvimento psicossocial do adolescente (Moffitt, 1993; Dias, Conde, Formiga, \& Gonçalves, 2014; Neumann, Hare, \& Pardini, 2014; Formiga, Duarte e outros, 2015; Morizot \& Kazemian, 2015).

A escala de condutas antissociais e delitivas é destinada para a medida do comportamento juvenil desviante e tem sido utilizada em distintas amostras e países (Sintra, Lopes, \& Formiga, 2011; Formiga, Duarte e outros, 2015; Formiga, Aguiar, Estevam, \& Omar, 2016). Devido à gravidade e constância desse fenômeno na contemporaneidade ele ainda é alvo de insistente investigação, especialmente com amostras de adolescentes, pois frente aos processos psicossociais desenvolvimentistas tal grupo possui aspectos inerentes ao seu comportamento, que são bem peculiares à dinâmica similar do desvio de conduta e que têm surgido em diferentes fases do desenvolvimento, podendo diminuir ao longo do processo de desenvolvimento social (Kazdin \& Buela-Casal, 1998).

Apesar de se encontrar instrumentos psicológicos que avaliam, de forma específica, o fenômeno da conduta desviante em jovens, a partir de uma perspectiva social ou através do transtorno psíquico (Born \& Bradley, 2005), no Brasil uma medida psicológica que vem sendo apresentada como capaz de avaliar as condutas desviantes em jovens é a Escala de Condutas Antissociais e Delitivas (ECAD). Trata-se de um instrumento de medida atitudinal descritiva e preditiva do comportamento delinquente, $o$ qual foi desenvolvido por Seisdedos (1988) e validado por Formiga \& Gouveia (2003) para o contexto brasileiro e vem revelando indicadores psicométricos confiáveis relacionados à fidedignidade da escala em distintas amostras brasileiras (Formiga, 2003), portuguesas (Formiga, Duarte e outros, 2015) e argentinas (Formiga, Aguiar e outros, 2016) e que apresenta-se fatorialmente organizado em dois tipos de comportamentos socialmente desviantes: os antissociais e os delitivos. Para os autores supracitados, tais condutas interferem nos direitos e nos deveres das pessoas, ameaçando o seu bem-estar social e individual: "o primeiro tipo (antissociais) refere-se aos comportamentos que desafiam a ordem social, enquanto o segundo tipo (delitivos) integram comportamentos considerados como estando à margem da lei, caracterizando uma infração ou uma conduta faltosa e prejudicial a alguém ou mesmo à sociedade como um todo" (Formiga, Duarte e outros, 2015, p. 719).

A importância sobre as pesquisas que caracterizam um continuиm teórico- 


\section{DELITIVAS EM JOVENS}

empírico desenvolvido a respeito da testagem psicológica, relacionando o tema da conduta desviante e sua medida entre os jovens, se concentra na qualificação de um instrumento que contemple uma dinâmica teórica e aplicada destinada às pesquisas básicas e interventivas na área da ciência humana e social. Para isso, é importante não apenas uma condição teórica e metodológica mas também matemática, que seja capaz de revelar, estatisticamente, indicadores psicométricos que afirmem uma medida e sua teoria adequadas, que favoreça preditivamente a compreensão do comportamento na relação indivíduosociedade.

É no contexto científico destacado no parágrafo acima que se inclui a análise desenvolvida por Formiga (2003) e Formiga \& Gouveia (2003). Estes autores apontam que um dos objetivos das técnicas multivariadas para a verificação fatorial e de confiabilidade das medidas psicológicas é expandir a habilidade do pesquisador em explorar e ser eficiente na estatística utilizada na modelagem da estrutura teórica hipotetizada, condição que possibilita relacionar de forma simultânea as dependências entre as variáveis e seus erros de medidas. Dessa forma, o interesse do presente estudo tem as seguintes direções: 1 - refere-se, ainda, à escassez de instrumentos de medida da delinquência juvenil adaptados à população juvenil brasileira; 2 - devido às orientações teóricas elencadas por Formiga, Duarte e outros, 2015), as quais foram sugeridas para futuros estudos (por exemplo, verificar a estrutura da ECAD em outros contextos sociais e intraculturais e avaliar a consistência dessa medida em termos da faixa etária, sexo, inserção educacional, etc.) e; 3 - reunir características de avaliação do comportamento desviante favorável à ECAD ao longo do tempo e em distintos contextos. Assim, este estudo tem como principal objetivo comprovar a organização da estrutura bifatorial da Escala de Condutas Antissociais e Delitivas - ECAD - em jovens de Montes Claros/MG e avaliar as diferenças dessa medida em função de variáveis sociodemográficas.

\section{Método}

A presente pesquisa é considerada do tipo quantitativo, transversal e correlacional.

\subsection{Amostra}

A amostra foi constituidas por 691 sujeitos da cidade de Montes Claros-MG, distribuídos no nível educacional fundamental (47\%) e no nível médio (57\%), sendo $52 \%$ do sexo masculino e $48 \%$ de sexo 
feminino, com idades compreendidas entre os 12 e $19 \operatorname{anos}(\mathrm{M}=14,64, \mathrm{DP}=1,79 ; \mathrm{Mo}=$ 15). A maioria (33\%) tinha uma renda econômica que variava de 724,00 a 1.000,00 reais. A amostragem foi não probabilística e intencional, pois buscava garantir os resultados com base na faixa etária salientada pela literatura desenvolvimentista quanto à intensidade do jovem apresentar a conduta desviante.

Para estabelecer a significância da amostra, esta foi calculada no pacote estatístico G Power 3.1, que é um software destinado a calcular o poder estatístico - o teste de hipótese -, tendo como base não apenas o ' $\mathrm{n}$ ' necessário para a pesquisa, mas também o tipo de cálculo a ser realizado (Faul, Erdfelder, Lang, \& Buchner, 2009). Para a coleta de dados deste estudo, foi considerada uma probabilidade de $95 \%$ ( $\mathrm{p}$ < $0,05)$, uma magnitude do efeito amostral $(r \geq$ $0,30)$ e um padrão de poder hipotético $(\pi \geq$ $0,80)$. A partir desses critérios, uma amostra mínima de 400 sujeitos revelou-se suficiente, com indicadores: $\mathrm{t} \geq 1,98 ; \pi \geq 0,99 ; \mathrm{p}<0,05$ ).

\subsection{Instrumento}

Escala de Condutas Antissociais e Delitivas - ECAD, de Seisdedos (1988) -, adaptado para a população brasileira por Formiga (2003) e Formiga \& Gouveia
(2003): a ECAD é uma medida que avalia a conduta desviante em jovens e é composta por 40 itens, com 20 deles pertencentes ao fator da conduta antissocial e 20 itens à conduta delitiva. A primeira refere-se aos comportamentos que desafiam a ordem social (exemplos: jogar lixo no chão mesmo quando há perto um cesto de lixo; tocar a campainha na casa de alguém e sair correndo), enquanto a segunda diz respeito a comportamentos considerados à margem da lei, caracterizando uma infração ou uma conduta faltosa e prejudicial a alguém ou mesmo à sociedade como um todo (exemplos: roubar objetos dos carros; conseguir dinheiro ameaçando pessoas mais fracas). Para cada item, os respondentes deveriam indicar, em uma escala de Likert de 0 a $9(0=$ Nunca e $9=$ Sempre), a frequência do comportamento assinalado.

$\mathrm{O}$ presente instrumento foi adaptado e validado por Formiga \& Gouveia (2003) e Formiga (2003) para a população brasileira, revelando indicadores psicométricos muito bons para as dimensões das Condutas Antissociais e Delitivas. Além dos autores observarem alfas de Cronbach acima de 0,70 para as duas dimensões e em relação à Análise Fatorial Confirmatória, os indicadores psicométricos $\left(\chi^{2} / \mathrm{gl}=1.35\right.$; 


\section{DELITIVAS EM JOVENS}

AGFI $=0.89 ;$ PHI $(\Phi)=0.79, \mathrm{p}>.05)$ comprovaram a estrutura fatorial pretendida.

\subsection{Caracterização sociodemográfica}

Além da ECAD, um questionário de caracterização sociodemográfica foi incluído para avaliar a caracterização dos/as participantes deste estudo bem como realizar um controle estatístico de atributos que possam interferir diretamente nos resultados.

\subsection{Procedimentos da coleta de dados e} éticos

$\mathrm{O}$ instrumento da pesquisa foi administrado aos sujeitos do ensino fundamental e médio nas instituições públicas na cidade de Montes Claros-MG, em sala de aula. As pessoas que mostraram interesse e que deram o seu consentimento em participar neste estudo foram conscientizadas de que não havia respostas certas ou erradas, que respondessem de acordo com o que pensavam.

A todos os jovens foi assegurado o anonimato das suas respostas e lhes garantido que as mesmas seriam tratadas em seu conjunto. Dessa forma, contando com as instruções necessárias para que o questionário pudesse ser respondido, os pesquisadores estiveram presentes durante toda a aplicação para o esclarecimento das dúvidas que surgissem. Um tempo médio de 50 minutos foi o suficiente para concluir essa atividade.

Todos os procedimentos adotados nesta pesquisa seguiram as orientações previstas na Resolução CNS 466/12, entre outras normas expedidas pelo Conselho Nacional da Saúde (CNS), bem como da Resolução 016/2012 do Conselho Federal de Psicologia. O projeto de pesquisa foi submetido a um Comitê de Ética, no qual obteve a aprovação através do Parecer Consubstanciado de Número 798.135, datado de 19/09/2014.

\subsection{Análise dos dados}

Para a análise dos dados deste estudo, utilizou-se a versão 21.0 do programa estatístico SPSS para Windows. Foram computadas estatísticas descritivas (medidas de tendência central e dispersão) e MANOVA. Além destes cálculos, realizou-se uma análise fatorial confirmatória com recurso ao programa estatístico AMOS Grafics 21.0, com o intuito de se avaliar a consistência estrutural do modelo já previamente encontrado para a ECAD. Considerou-se como entrada a matriz de covariâncias, tendo sido adotado o estimador ML (Maximum Likelihood).

Uma vez que esta análise estatística é mais criteriosa e rigorosa, testou-se a 
estrutura teórica quanto ao modelo bifatorial. Esta análise apresenta alguns índices que permitem avaliar a qualidade de ajuste do modelo proposto (Byrne, 1989; Joreskög \& Sörbom, 1989; Van de Vijver \& Leung, 1997; Hair, Anderson, Tatham, \& Black, 2009). Para tanto, utilizou-se: o teste do $\chi^{2}$ (quiquadrado), que testa a probabilidade do modelo teórico se ajustar aos dados; a Raiz Quadrada Média Residual Saturado $\left(\mathrm{RMR}_{\mathrm{st}}\right)$, que indica o ajustamento do modelo teórico aos dados, na medida em que a diferença entre os dois se aproxima de zero; $o$ Goodness-of-Fit Index (GFI) e o Adjusted Goodness-of-Fit Index (AGFI) são análogos ao $\mathrm{R}^{2}$ em regressão múltipla e indicam a proporção de variância-covariância nos dados explicada pelo modelo; o Comparative Fit Index (CFI) de uma forma geral compara o modelo estimado e o modelo nulo; o Tucker-Lewis Index (TLI) apresenta uma medida de parcimônia entre os índices do modelo proposto e do modelo nulo; o RootMean-Square Error of Approximation (RMSEA) é considerado um indicador de "maldade" de ajuste, isto é, valores altos indicam um modelo não ajustado; o Expected Cross-Validation Index (ECVI) e o Consistent Akaike Information Criterion (CAIC) são indicadores geralmente empregados para avaliar a adequação de um modelo determinado em relação a outro. Valores baixos do ECVI e CAIC expressam o modelo com melhor ajuste; Akaike's Information Criteria (AIC) é um critério que utiliza a parcimônia na avaliação do modelo levando em conta o número de parâmetros estimados. É usado quando são comparados dois ou mais modelos; o Browne-Cudeck Criterion (BCC) é um critério que funciona da mesma maneira que o AIC e o CAIC, com a diferença de que este impõe grandes penalidades para a complexidade do modelo; Bayes Information Criterion (BIC) mostra-se de forma mais consistente, uma vez que cada ajuste realizado nos componentes explicativos é gerado com base no ajuste adequado dos dados, penalizando severamente modelos com muitos parâmetros (Byrne, 1989; Joreskög \& Sörbom, 1989; Van de Vijver \& Leung, 1997; Hair e outros, 2009).

Além desses cálculos, foi realizado ainda o cálculo de Confiabilidade Composta (CC) quanto à Variância Média Extraída (VME). No primeiro indicador exige-se que o nível do escore seja acima de 0,70, enquanto no segundo indicador é preciso um nível acima de 0,50 .

\section{Resultados}




\section{DELITIVAS EM JOVENS}

A fim de atender à hipotese oblíquo (com os dois fatores

apresentada, gerou-se no programa estatístico

AMOS 21.0 a proposta bifatorial da medida pretendida, a qual foi previamente estabelecida por Formiga (2003) e Formiga \& Gouveia (2003). Foram gerados também os modelos comparativos, como o unifatorial e o ortogonal, este com dois fatores não relacionados, a fim de compará-los ao modelo interdependentes), o qual se espera ser comprovado. Os resultados revelaram que, quando se comparou o modelo hipotetizado aos demais modelos estabelecidos, o modelo oblíquo bifatorial foi o que revelou melhores indicadores psicométricos para a estrutura fatorial esperada (Tabela 1).

Tabela 1

Indicadores psicométricos da comparação da estrutura fatorial da ECAD

\begin{tabular}{|c|c|c|c|c|c|c|c|c|}
\hline \multirow{2}{*}{ Modelos } & \multicolumn{3}{|c|}{$\begin{array}{c}\text { Medidas de ajuste } \\
\text { absoluto }\end{array}$} & \multicolumn{3}{|c|}{$\begin{array}{l}\text { Medidas de ajuste } \\
\text { incremental }\end{array}$} & \multicolumn{2}{|c|}{$\begin{array}{c}\text { Medidas de ajuste } \\
\text { parcimonioso }\end{array}$} \\
\hline & $\chi^{2} / g 1$ & GFI & AGFI & CFI & TLI & $\begin{array}{c}\text { RMSEA } \\
\text { (intervalo) }\end{array}$ & CAIC & $\begin{array}{c}\text { ECVI } \\
\text { (intervalo) }\end{array}$ \\
\hline Modelo $1 *$ & 3,62 & 0,78 & 0,76 & 0,12 & 0,7 & $\begin{array}{c}0,07 \\
(0,06-0,07)\end{array}$ & 3275,42 & $\begin{array}{c}4,6 \\
(4,34-4,86)\end{array}$ \\
\hline Modelo $2 * *$ & 7,29 & 0,67 & 0,63 & 0,72 & 0,71 & $\begin{array}{c}0,1 \\
(0,09-0,1)\end{array}$ & 5985,19 & $\begin{array}{c}8,98 \\
(8,61-9,36)\end{array}$ \\
\hline Modelo $3 * * *$ & 1,88 & 0,94 & 0,91 & 0,93 & 0,92 & $\begin{array}{c}0,04 \\
(0,03-0,05)\end{array}$ & 2963,75 & $\begin{array}{c}2,55 \\
(2,4-2,7)\end{array}$ \\
\hline
\end{tabular}

Notas: *Modelo unifatorial; **Modelo bifatorial ortogonal; ***Modelo bifatorial oblíquo.

Considerando os indicadores apresentados anteriormente, destaca-se que todas as saturações (Lambdas, $\lambda$ ) estiveram não somente dentro do intervalo esperado $\mid 0$ 1|, mas foram estatisticamente diferentes de zero $(t>1,96, p<0,05)$, revelando a não existência de problemas da estimação proposta na medida em questão, as quais foram superiores a zero e não ultrapassaram o parâmetro considerado limite, igual a 1 (cf.
Tabela 2). Tais resultados corroboram a existência do modelo bifatorial com interdenpendencias entre os fatores, destinado a mensurar as condutas desviantes (condutas antissociais e delitivas) nos jovens na cidade de Montes Claros-MG que participaram das pesquisas, revelando uma associação Phi $(\varphi)$ positiva entre os fatores $(\lambda$ $=0.88)$. 
MARIA DE FATIMA DE MATOS MAIA, NILTON SOARES FORMIGA, THATIANA MAIA TOLENTINO, CELINA APARECIDA GONÇALVES LIMA, BERENILDE VALÉRIA DE OLIVEIRA SOUSA

Tabela 2

Estrutura Fatorial da ECAD em jovens de Montes Claros-MG

\begin{tabular}{|c|c|c|c|c|c|}
\hline$\xi$ (construto) & $\begin{array}{c}\chi \text { (variáveis) } \\
\text { [itens] }\end{array}$ & $\lambda$ & $\varepsilon($ erros $)$ & $\mathrm{CC}$ & VME \\
\hline \multirow{20}{*}{ Conduta Antissocial } & ECAD1 & 0,50 & 0,25 & \multirow{20}{*}{0,93} & \multirow{20}{*}{0,67} \\
\hline & ECAD2 & 0,60 & 0,30 & & \\
\hline & ECAD4 & 0,66 & 0,31 & & \\
\hline & ECAD5 & 0,62 & 0,33 & & \\
\hline & ECAD9 & 0,69 & 0,41 & & \\
\hline & ECAD10 & 0,41 & 0,18 & & \\
\hline & ECAD11 & 0,67 & 0,42 & & \\
\hline & ECAD12 & 0,62 & 0,36 & & \\
\hline & ECAD15 & 0,70 & 0,43 & & \\
\hline & ECAD18 & 0,72 & 0,52 & & \\
\hline & ECAD21 & 0,61 & 0,37 & & \\
\hline & ECAD24 & 0,57 & 0,32 & & \\
\hline & ECAD26 & 0,43 & 0,18 & & \\
\hline & ECAD28 & 0,62 & 0,38 & & \\
\hline & ECAD29 & 0,65 & 0,42 & & \\
\hline & ECAD30 & 0,65 & 0,35 & & \\
\hline & ECAD34 & 0,66 & 0,42 & & \\
\hline & ECAD35 & 0,55 & 0,42 & & \\
\hline & ECAD37 & 0,62 & 0,44 & & \\
\hline & ECAD38 & 0,60 & 0,30 & & \\
\hline \multirow{20}{*}{ Conduta Delitiva } & ECAD3 & 0,59 & 0,52 & \multirow{20}{*}{0,95} & \multirow{20}{*}{0,71} \\
\hline & ECAD6 & 0,53 & 0,39 & & \\
\hline & ECAD7 & 0,69 & 0,64 & & \\
\hline & ECAD8 & 0,57 & 0,59 & & \\
\hline & ECAD13 & 0,44 & 0,22 & & \\
\hline & ECAD14 & 0,66 & 0,56 & & \\
\hline & ECAD16 & 0,81 & 0,78 & & \\
\hline & ECAD17 & 0,81 & 0,82 & & \\
\hline & ECAD 19 & 0,75 & 0,73 & & \\
\hline & ECAD20 & 0,76 & 0,69 & & \\
\hline & ECAD22 & 0,83 & 0,86 & & \\
\hline & ECAD23 & 0,86 & 0,85 & & \\
\hline & ECAD25 & 0,82 & 0,83 & & \\
\hline & ECAD27 & 0,72 & 0,84 & & \\
\hline & ECAD31 & 0,85 & 0,82 & & \\
\hline & ECAD32 & 0,81 & 0,75 & & \\
\hline & ECAD33 & 0,73 & 0,68 & & \\
\hline & ECAD36 & 0,63 & 0,60 & & \\
\hline & ECAD39 & 0,82 & 0,76 & & \\
\hline & ECAD40 & 0,60 & 0,67 & & \\
\hline
\end{tabular}

Notas: $\lambda=$ Escores fatoriais da estrutura; $\varepsilon$ (erros) $=$ Erros de medida da estrutura; $\chi=$ variáveis (itens); $\xi=$ construto psicológico; VME = Variância Média Extraída; $\mathrm{CC}=$ Confiabilidade Composta. 


\section{DELITIVAS EM JOVENS}

Além dos indicadores psicométricos observados na Tabela 1, outros mais parcimoniosos (por exemplo: o AIC, BIC e BCC) garantiram o modelo hipotetizado, justamente por serem resultados que acompanham o CAIC e ECVI (estes são indicadores que considerando os ajustes realizados para o modelo não foi exagerado e condizem com a proposta teórica), os quais servem para avaliar a adequação de um modelo determinado em relação a outros modelos para comparação da melhor estrutura fatorial, neste caso a proposta do modelo bifatorial oblíquo em relação ao modelo bifatorial ortogonal e unifatorial. $\mathrm{O}$ modelo proposto revelou melhores resultados em relação aos outros modelos (ver Tabela 3).

Tabela 3

Indicadores psicométricos de parcimônia para a comparação da estrutura fatorial da ECAD

\begin{tabular}{lccc}
\hline \multirow{2}{*}{ Modelos } & \multicolumn{3}{c}{ Indicador de parcimônia } \\
\cline { 2 - 4 } & AIC & BIC & BCC \\
\hline Modelo 1* & 3841,17 & 4195,42 & 4852,54 \\
Modelo 2** & 5550,94 & 5905,19 & 5562,31 \\
Modelo 3*** & 2810,59 & 3169,26 & 2822,10 \\
\hline
\end{tabular}

Notas: *Modelo unifatorial; **Modelo bifatorial ortogonal; ***

Modelo bifatorial oblíquo.

Com o objetivo de verificar a validade do construto em questão, realizou-se o cálculo de Confiabilidade Composta, o qual exige que o nível do escore seja acima de 0,70 e o da Variância Média Extraída neste é preciso um nível acima de 0,50 (Fornell \& Larcker, 1981; Hair e outros, 2009). Observou-se que para as dimensões das condutas antissociais e delitivas, o $\mathrm{CC}$ e o VME estiveram acima do exigido na literatura, evidenciando a confiabilidade e validade convergente do construto utilizado e segurança fatorial para a população juvenil (cf. Tabela 1). Sendo essa escala administrada em outros contextos brasileiros, resolveu-se considerar mais um indicador psicométrico para que sustentasse a segurança na qualidade da medida ECAD. Para isso, realizou-se o cálculo de correlação intraclasse e este apresentou: para a escala total [CAD] um ICC $=0,95($ IC $95 \%=0,94-0,96 ; \mathrm{p}<0,05) ;$ para a dimensão da conduta antisssocial observouse um ICC $=0,91$ (IC 95\% =0,90-0,93; p < 0,05); e para a dimensão da conduta delitiva o ICC foi de 0,94 (IC 95\% = 0,93-0,95; p < 0,05), condição essa que torna o teste fidedigno desde a última aplicação no estudo de Formiga e outros (2015). 
MARIA DE FATIMA DE MATOS MAIA, NILTON SOARES FORMIGA, THATIANA MAIA TOLENTINO, CELINA APARECIDA GONÇALVES LIMA, BERENILDE VALÉRIA DE OLIVEIRA SOUSA

Além de se observar uma associação Phi (Ф) entre os fatores com boa força associativa e na relação item-fator, tal resultado foi confirmado quando foram realizadas as estimativas de predição a partir da análise de regressão, a qual se revelou para o modelo hipotetizado através da identificação das variáveis que foram significativas e a razão critério que estiveram dentro do que é estatisticamente exigido $(t>$ $1.96, p<0,05)$. Esses resultados evidenciam ausência de uma multicolineariedade, condição essa observada no VIF (definida em

Tabela 4

Indicadores das estimativas preditivas entre itens-fatores da ECAD

\begin{tabular}{ccc|ccccc}
\hline Itens & $<---$ & Fator & Estimativa & DP & $\begin{array}{c}\text { Razão } \\
\text { Critério }\end{array}$ & $\mathrm{p}<$ & VIF \\
\hline CAS38 & $<---$ & CAS & 1,352 & 0,148 & 9,117 & 0,001 & 1,88 \\
CAS37 & $<---$ & CAS & 1,520 & 0,163 & 9,346 & 0,001 & 2,23 \\
CAS35 & $<---$ & CAS & 1,015 & 0,102 & 9,952 & 0,001 & 2,21 \\
CAS34 & $<---$ & CAS & 1,228 & 0,120 & 10,236 & 0,001 & 2,81 \\
CAS30 & $<---$ & CAS & 1,316 & 0,135 & 9,759 & 0,001 & 2,45 \\
CAS29 & $<--$ & CAS & 1,490 & 0,145 & 10,284 & 0,001 & 1,90 \\
CAS28 & $<---$ & CAS & 1,210 & 0,128 & 9,430 & 0,001 & 1,99 \\
CAS26 & $<---$ & CAS & 1,153 & 0,139 & 8,292 & 0,001 & 1,62 \\
CAS24 & $<---$ & CAS & 1,153 & 0,125 & 9,235 & 0,001 & 1,93 \\
CAS21 & $<---$ & CAS & 1,290 & 0,138 & 9,327 & 0,001 & 1,85 \\
CAS18 & $<---$ & CAS & 1,284 & 0,120 & 10,693 & 0,001 & 2,97 \\
CAS15 & $<--$ & CAS & 1,491 & 0,140 & 10,641 & 0,001 & 2,45 \\
CAS12 & $<---$ & CAS & 1,165 & 0,118 & 9,905 & 0,001 & 2,52 \\
CAS11 & $<---$ & CAS & 1,204 & 0,106 & 11,332 & 0,001 & 2,03 \\
CAS10 & $<---$ & CAS & 0,750 & 0,093 & 8,030 & 0,001 & 2,50 \\
CAS9 & $<---$ & CAS & 0,955 & 0,093 & 10,264 & 0,001 & 1,52 \\
CAS5 & $<---$ & CAS & 1,115 & 0,109 & 10,251 & 0,001 & 1,81 \\
CAS4 & $<---$ & CAS & 1,210 & 0,131 & 9,266 & 0,001 & 1,87 \\
CAS2 & $<---$ & CAS & 1,154 & 0,105 & 10,967 & 0,001 & 2,82 \\
CAS1 & $<---$ & CAS & 1,000 & --- & --- & --- & 1,60 \\
CD3 & $<---$ & CD & 1,000 & --- & --- & --- & 2,29 \\
\hline
\end{tabular}




\begin{tabular}{ccc|ccccc}
\hline Itens & $<---$ & Fator & Estimativa & DP & $\begin{array}{c}\text { Razão } \\
\text { Critério }\end{array}$ & $\mathrm{p}<$ & VIF \\
\hline CD6 & $<---$ & CD & 0,537 & 0,039 & 13,620 & 0,001 & 1,91 \\
CD7 & $<---$ & CD & 0,834 & 0,051 & 16,448 & 0,001 & 2,84 \\
CD8 & $<---$ & CD & 0,871 & 0,056 & 15,462 & 0,001 & 2,72 \\
CD13 & $<---$ & CD & 0,785 & 0,082 & 9,525 & 0,001 & 1,51 \\
CD14 & $<---$ & CD & 1,280 & 0,085 & 15,011 & 0,001 & 2,35 \\
CD16 & $<---$ & CD & 1,067 & 0,066 & 16,232 & 0,001 & 4,07 \\
CD17 & $<--$ & CD & 1,094 & 0,070 & 15,714 & 0,001 & 4,62 \\
CD19 & $<---$ & CD & 0,998 & 0,060 & 16,494 & 0,001 & 3,55 \\
CD20 & $<---$ & CD & 1,193 & 0,075 & 15,831 & 0,001 & 3,08 \\
CD22 & $<---$ & CD & 1,006 & 0,067 & 14,903 & 0,001 & 3,17 \\
CD23 & $<---$ & CD & 0,915 & 0,058 & 15,903 & 0,001 & 3,11 \\
CD25 & $<---$ & CD & 0,891 & 0,062 & 14,367 & 0,001 & 3,35 \\
27 & $<---$ & CD & 0,924 & 0,069 & 13,427 & 0,001 & 4,32 \\
CD31 & $<---$ & CD & 1,164 & 0,070 & 16,543 & 0,001 & 4,48 \\
CD32 & $<---$ & CD & 0,883 & 0,057 & 15,415 & 0,001 & 3,89 \\
CD33 & $<---$ & CD & 1,246 & 0,088 & 14,091 & 0,001 & 3,02 \\
CD36 & $<---$ & CD & 1,409 & 0,100 & 14,039 & 0,001 & 2,51 \\
CD39 & $<---$ & CD & 1,173 & 0,075 & 15,572 & 0,001 & 3,83 \\
CD40 & $<---$ & CD & 1,604 & 0,122 & 13,169 & 0,001 & 3,05 \\
\hline
\end{tabular}

A partir desses resultados é possível destacar a garantia da organização fatorial do modelo pretendido, avaliador das condutas desviantes em jovens. A medida em questão evidencia que as condutas medidas referemse aos comportamentos que desafiam a ordem social ou que estão à margem da lei, salientadas nas associações itens-fator, podendo ser avaliadas a partir dos 40 itens distribuídos em duas dimensões: conduta antissocial e conduta delitiva, convergindo em direção aos resultados nos estudos preliminares com a ECAD (Formiga, 2003; Formiga \& Gouveia, 2003), bem como para os mais recentes com amostras de outros países: Portugal (Formiga, Duarte e outros,
2015) e Argentina (Formiga, Aguiar e outros, 2016).

Mesmo confirmada a estrutura fatorial da ECAD, procurou-se comparar, a partir de uma MANOVA, as pontuações médias das respostas dos sujeitos nas dimensões da ECAD, considerando as variáveis do sexo, idade e nível educacional escolar, evidenciaram resultados significativos apenas para as Condutas Delitivas (CD) no efeito principal com o nível escolar, sexo e idade e, para efeito de interação, o nível escolar versus idade e na conduta desviante (CAD pontuação total de todos os itens da ECAD) para o efeito principal na variável sexo e nível escolar (ver Tabela 5). Pode-se observar que 
MARIA DE FATIMA DE MATOS MAIA, NILTON SOARES FORMIGA, THATIANA MAIA TOLENTINO, CELINA APARECIDA GONÇALVES LIMA, BERENILDE VALÉRIA DE OLIVEIRA SOUSA

os homens apresentaram maiores escores do que as mulheres na conduta delitiva. Em relação à idade, os sujeitos mais velhos também pontuaram mais alto do que os demais níveis etários. Quanto ao nível escolar, os sujeitos do fundamental apresentaram maiores escores do que os sujeitos do nível médio. Por fim, em relação à interação entre nível escolar e idade, os sujeitos acima de 15 anos que estão no nível fundamental apresentaram escores superiores em relação às demais associações.
Ainda na Tabela 5 também poderá se observar os resultados significativos para a conduta antissocial e delituosa (CAD somatório de todos os itens da ECAD, categorizando como condutas desviantes). Apenas as variáveis sexo e nível escolar apresentaram diferenças nos escores. Respectivamente, na primeira os homens tiveram escores maiores do que as mulheres e na segunda os sujeitos do nível fundamental pontuaram mais alto na $\mathrm{CAD}$.

Tabela 5

Análise multivariada entre a conduta desviante em função das variáveis sociodemográficas

\begin{tabular}{|c|c|c|c|c|c|c|c|c|}
\hline \multirow{3}{*}{\multicolumn{2}{|c|}{ Variáveis Dependente }} & \multirow{2}{*}{\multicolumn{3}{|c|}{$\begin{array}{c}\text { Variável Independente } \\
\text { Conduta Delituosa }\end{array}$}} & \multirow{2}{*}{\multicolumn{4}{|c|}{ Estatística }} \\
\hline & & & & & & & & \\
\hline & & Média & $\mathrm{DP}$ & IC 95\% & $\mathrm{F}$ & $\mathrm{PO}$ & $\lambda$ Wilk's & $\mathrm{p}<$ \\
\hline \multirow{2}{*}{ Sexo } & Homem & 16,78 & 2,82 & $11,24-22,33$ & \multirow{2}{*}{17,08} & \multirow[b]{2}{*}{1} & \multirow{2}{*}{0,98} & \multirow{2}{*}{0,001} \\
\hline & Mulher & 3,75 & 1,17 & $1,44-6,05$ & & & & \\
\hline \multirow{3}{*}{ Idade } & 11-13 anos & 5,76 & 1,39 & $3,03-8,50$ & \multirow{3}{*}{12,61} & \multirow{3}{*}{0,82} & \multirow{3}{*}{0,98} & \multirow{3}{*}{0,01} \\
\hline & 14-16 anos & 7,13 & 1,14 & $4,89-9,37$ & & & & \\
\hline & 17-19 anos & 19,62 & 4,63 & $10,52-26,70$ & & & & \\
\hline \multirow{3}{*}{$\begin{array}{l}\text { Nível } \\
\text { Escolar }\end{array}$} & Fundamental & 15,49 & 2,84 & $9,92-21,07$ & \multirow{2}{*}{11,43} & \multirow{2}{*}{0,87} & \multirow{2}{*}{0,98} & \multirow{2}{*}{0,01} \\
\hline & Médio & 5,36 & 1,11 & $3,17-7,55$ & & & & \\
\hline & F. 11-13 anos & 5,76 & 1,39 & $3,03-8,5$ & \multirow{6}{*}{7,02} & \multirow{6}{*}{0,65} & \multirow{6}{*}{0,99} & \multirow{6}{*}{0,05} \\
\hline \multirow{5}{*}{$\begin{array}{l}\text { Nível } \\
\text { Escolar } \\
\text { versus } \\
\text { Idade }\end{array}$} & F. 14-16 anos & 9,72 & 1,89 & $6,01-13,42$ & & & & \\
\hline & F. 17-19 anos & 46,5 & 13,4 & 20,19-72,81 & & & & \\
\hline & M. 11-13 anos & --- & --- & --- & & & & \\
\hline & M. 14-16 anos & 4,55 & 1,28 & $2,03-7,06$ & & & & \\
\hline & M. 17-19 anos & 6,17 & 1,82 & $2,6-9,75$ & & & & \\
\hline & & \multicolumn{3}{|c|}{ Conduta Antissocial e Delitiva } & \multicolumn{4}{|c|}{ Estatística } \\
\hline \multirow{2}{*}{ Sexo } & Homem & 42,88 & 6,47 & $30,17-55,6$ & \multirow{2}{*}{4,08} & \multirow{2}{*}{0,52} & \multirow{2}{*}{0,98} & \multirow{2}{*}{0,05} \\
\hline & Mulher & 21,11 & 2,69 & $19,81-30,19$ & & & & \\
\hline Nível & Fundamental & 42,34 & 2,51 & $29,45-55,03$ & \multirow{2}{*}{4,41} & \multirow{2}{*}{0,56} & \multirow{2}{*}{0,98} & \multirow{2}{*}{0,05} \\
\hline Escolar & Médio & 25,9 & 2,53 & $20,89-30,91$ & & & & \\
\hline
\end{tabular}

Notas: $\mathrm{H}=$ homem, $\mathrm{M}=$ mulher, $\mathrm{F}=$ nível fundamental, $\mathrm{M}=$ nível médio. 


\section{DELITIVAS EM JOVENS}

\section{Discussão}

Considerando os achados neste estudo, corrobora-se a estrutura fatorial composta por dois fatores, os quais, hierarquicamente, se organizaram na dimensão da conduta antissocial e delitiva. Estes resultados revelaram uma consistência da perspectiva teórica abordada por Formiga \& Gouveia (2003), Rocha, Formiga, \& Lopes (2011), Duarte, Santos, Cruz, \& Grangeia (2015), Formiga, Duarte e outros (2015) e Formiga, Souza e outros (2016). Esses autores consideram que tais condutas referem-se à frequência e à intensidade de condutas referentes à infração das normas sociais e leis socialmente aceitas, as quais podem ser consideradas como condutas antissociais e delitivas, sendo as delitivas conforme Rocha e outros (2011) e Duarte e outros (2015) vinculadas às infrações mais graves, pois visam a causar prejuízos de uma maneira direta a pessoas, bem como prejuízo material e social da vítima no entorno interpessoal de quem é vitimado.

No entanto, Formiga, Souza e outros (2016) colocam que eles não possuem a intenção nem o interesse em avaliar um transtorno psiquiátrico, mas considerar uma perspectiva psicológica e social das condutas desviantes dos jovens. Para esses autores convém considerar desde a não aceitação das normas sociais como também a aceitação de pessoas, assim como o furto, a formação de gangues, o uso de drogas lícitas ou ilícitas e o uso de armas.

Com base nos estudos, no Brasil, com o mesmo construto, independente da amostra em distintos contextos geopolíticos, a referida escala (ECAD) apresenta uma consistência empírica e, com base nos diversos indicadores psicométricos, o modelo previamente proposto se mantém em sua bifatorialidade.

A observação de associações lambdas $(\lambda)$ entre os fatores apresentaram boa força associativa entre elas (cf. Tabela 2), sendo confirmada pela observação das estimativas de predição do modelo proposto, a partir da análise de regressão (cf. Tabela 4). Com isso, garante-se o modelo bifatorial, o qual é capaz de mensurar a conduta desviante, principalmente a sua forma da antissociabilidade e delituosidade. A primeira forma diz respeito a não conscientização das normas que devem ser respeitadas, às travessuras dos jovens ou simplesmente à busca de romper com algumas leis sociais. Esse tipo de conduta caracteriza-se pelo fato de incomodarem, mas sem que causem, necessariamente, danos físicos às outras pessoas (Formiga, 2002; 2003; Formiga \& 
Gouveia, 2003). Portanto, àquele que de maneira natural tende a não viver em sociedade ou a presentar dificuldades no trato com o outro e assim dificultar o relacionar-se. A segunda, provavelmente, originada da primeira, refere-se àquelas condutas merecedoras de punição, capazes de causar danos graves, morais e/ou físicos. Elas podem ser consideradas mais severas que as anteriores, representando uma ameaça eminente à ordem social vigente (Formiga \& Gouveia, 2003).

Nos resultados da MANOVA, esperava-se que os sujeitos se diferenciassem também na dimensão da conduta antissocial, algo que não ocorreu. Em estudos anteriores sobre esse construto com amostras brasileiras, portuguesas e argentinas foram encontradas diferenças significativas para esse tipo de conduta (Formiga \& Gouveia, 2003; Formiga, Duarte e outros, 2015; Formiga, Souza e outros, 2016). Contudo, nesta pesquisa os resultados significativos estiveram apenas na conduta delituosa e na desviante (somatório de todos os itens da escala ECAD).

De forma geral, os escores médios em relação aos homens e aos sujeitos mais velhos (faixa final da adolescência) corroboram os resultados dos autores supracitados referentes ao desvio de conduta. Um resultado distinto foi observado no presente estudo com relação ao nível escolar, pois o jovem do nível fundamental apresentou escore mais alto do que o jovem do nível médio. Nos estudos anteriores o resultado foi inversamente proporcional.

Outro resultado que chama atenção foi o efeito de interação. Os sujeitos mais velhos do nível escolar fundamental tiveram escores mais altos quando comparados aos demais (mais novos e mais velhos do nível médio), condição que permite refletir, considerando o contexto da pesquisa, que os respondentes estão se inserindo nas condutas desviantes mais cedo. Tal situação mostra que atualmente é grande a valorização do individualismo, justificado sobre um estigma de liberdade e democracia. Esse fato, mesmo objetivando ser livre e democrático, em termos de um comportamento sociopolítico, não impede o fato de rejeitar as regras sociais, o respeito aos direitos alheios e ao imperativo do dever; tais atitudes devem ser envolvidas de normas e considerações em relação ao comportamento socialmente desejável e não ser lesado psíquica e socialmente (Formiga \& Diniz, 2011).

\section{Considerações Finais}




\section{DELITIVAS EM JOVENS}

De forma geral, espera-se que os objetivos do presente estudo tenham sido cumpridos, principalmente, no que se refere à comprovação da medida de conduta desviante em adolescentes. Os resultados desta pesquisa revelaram não apenas a consistência temporal e contextual do instrumento em relação a sua estrutura fatorial mensurada para a amostra de jovens, distribuída em dois fatores: conduta antissocial e conduta delitiva, que se correlacionam positivamente, isto é, provavelmente aquele jovem que apresentar maior escore em uma dessas condutas terá escore alto na dimensão. Esses resultados, mais do que salientar uma proposta de avaliação psicológica da conduta desviante entre os jovens, vêm confirmar a relação construto-medida, proposta previamente por Formiga \& Gouveia (2003). Essa relação diz respeito aos comportamentos desafiadores da ordem social ou que não estão de acordo com os códigos estabelecidos pelas autoridades de determinado espaço geográfico, associados aos preceitos morais socialmente estabelecidos, infringindo simultaneamente códigos de conduta moral e social.

Apesar de esta pesquisa garantir o uso da escala para o contexto brasileiro, é também importante a realização de análises comparativas quanto à relação divergente e convergente, contemplando construtos distintos e/ou semelhantes. Tal condição teria como objetivo verificar a manutenção dos fatores e a relação específica com os itens que o compõem em termos da temporalidade, cultura e comportamento político, visando a uma avaliação entre fator e itens de forma mais segura quanto à característica de uma medida preditiva desse tipo de comportamento.

Por fim, com base nestes resultados, recomenda-se a atualização do instrumento por meio da inclusão de itens com foco na contemporaneidade, como a adaptação de itens que contemplam valores econômicos, comportamento interpessoal e outros mais próximos à realidade do jovem. Sugere-se ainda a releitura de itens como "pegar 10 reais na bolsa dos pais". Outra sugestão para oferecer melhor qualidade para escala estaria associada a um estudo sobre invariância da medida ECAD tanto para avaliar as diferenças entre os escores médios quanto dos indicadores psicométricos com distintas amostras. 


\section{Referências}

Born, R.T., \& Bradley, D.C. (2005). Structure And Function Of Visual Area Mt. Annual Review Of Neuroscience. 28,157-189. doi.org/10.1146/annurev.neuro.26.041002.131052

Byrne, B. M. (1989). A primer of LISREL: Basic applications and programming for confirmatory factor analytic models. New York: Springer - Verlag. doi.org/10.1007/978-1-4613-8885-2

Dias, J., Conde, R., Formiga, N., \& Abrunhosa G. Rui. (2014). International self-report delinquency (ISRD3): tradução e adaptação ao contexto cabo-verdiano. Liberabit, 20(2), 335351.

Duarte, V., Santos, M., Cruz, O., \& Grangeia, H. (2015). Delinquência Juvenil: Explicações e Implicações, Castêlo da Maia: Edições ISMAI.

Faul F., Erdfelder E., Buchner A., \& Lang A.-G. (2009). Statistical power analyses using G*Power 3.1: tests for correlation and regression analyses. Behavior Research Methods, 41, 1149-1160. doi.org/10.3758/BRM.41.4.1149

Formiga, N. S., \& Diniz, A. S. (2011). Estilo da Orientação Cultural e Condutas Desviantes: Testagem de um Modelo Teórico. Psicologia em Pesquisa, 5(1), 02-11.

Formiga, N. S., Duarte, V., Neves, S., Machado, M., \& Machado, F. (2015). Escala de condutas antissociais e delitivas: estrutura fatorial da versão portuguesa. Revista Reflexão e Crítica, 28 (4), 718-727. doi.org/10.1590/1678-7153.201528409

Formiga, N.S. (2002). Condutas antissociais e delitivas: uma explicação em termos de valores humanos. Dissertação de Mestrado não publicada. Universidade Federal da Paraíba. PB, Brasil.

Formiga, N.S. (2003). Fidedignidade da escala de condutas antissociais e delitivas ao contexto brasileiro. Psicologia em Estudo, 8(2), 133-138. doi.org/10.1590/S1413-73722003000200014

Formiga, N.S., \& Gouveia, V.V. (2003). Valores Humanos e condutas antissociais e delitivas. Psicologia: Teoria e Prática 7(2)-134-170.

Formiga, N.S., Souza, M.A., Estevam, I., \& Omar, A. (2016). Evidência empírica da Escala de Condutas Antissociais e Delitivas em adolescentes argentinos. Boletim Academia Paulista de Psicologia, 36-124-140.

Fornell, C., \& Larcker, D. (1981). Structural Equation Models with Unobservable Variables and Measurement Error: Algebra and Statistics. Journal of Marketing Research, 18(3), 382-388.

Perspectivas em Psicologia, Uberlândia, vol. 22, n. 2, pp. 150 - 168, Jul/Dez, 2018 - ISSN 2237-6917 
Hair, J. F., Anderson, R. E., Tatham, R. L., \& Black, W. (2009). Análise Multivariada de dados. Porto Alegre: Bookman. doi.org/10.1177/002224378101800313

Jager, M. E., Altermann, B., F., Perrone, C. M., Silva, S. S., \& Garcia, D. A. C. (2014). O adolescente no contexto da saúde pública brasileira: reflexões sobre o PROSAD. O adolescente no contexto da saúde pública brasileira: reflexões sobre o PROSAD. Psicologia em Estudo, 19(2), 211-221. https://dx.doi.org/10.1590/1413-737221567004

Joreskög, K., \& Sörbom, D. (1989). LISREL 7 user's reference guide. Mooresville: Scientific Software.

Kazdin, A. E., \& Buela-Casal, G. (1998). Conducta antisocial. Madrid: Pirâmide.

Maroco, J. (2010). Análise de equações estruturais. Lisboa, Portugal: Report Number.

Moffitt, T. E. (1993). Adolescence-limited and life-course-persistent antisocial behavior: A developmental taxonomy. Psychological Review, 100(4), 674-701. doi.org/10.1037/0033295X.100.4.674

Morgado, A. M., \& Vale-Dias, M. L. (2014). Adolescência e delinquência: variáveis significativas para a construção de um modelo explicativo. Psicologia, Saúde \& Doenças, 15(1), 277-291. doi.org/10.15309/14psd150122

Morizot J., \& Kazemian L. (2015) 1 Introduction: Understanding Criminal and Antisocial Behavior Within a Developmental and Multidisciplinary Perspective. In: Morizot J., Kazemian L. (eds) The Development of Criminal and Antisocial Behavior. Springer, Cham. doi.org/10.1007/9783-319-08720-7_1

Negreiros, J. (2008). Delinquências juvenis: trajectórias, intervenções e prevenção. Porto: Livpsic.

Neumann, C. S., Hare, R. D., \& Pardini, D. A. (2015), Antisociality and the Construct of Psychopathy: Data From Across the Globe. Journal Personality, 83, 678-692. doi.org/10.1111/jopy.12127

Rocha, M. C. O., Formiga, N. S., \& Lopes, E. J. (2013). Una medida de la conducta desviada: La verificación empírica de estrutura fatorial de reclusos em Brasil. Eureka - Revista de investigação científica em psicologia, 10(2), 164-178.

Seisdedos, N. C. (1988). Cuestionario A-D de condutas antissociais-delictivas. Madri: TEA.

Silva, A.P., \& Salles, J.M.F. (2010). A violência na escola: abordagens teóricas e propostas de prevenção. Educar em Revista, 2, 217-232. https://dx.doi.org/10.1590/S010440602010000500013

Sintra,C. I. F., Lopes, P., \& Formiga, N. S. (2011). Condutas antissociais e delitivas e habilidades sociais em contexto forense. Psicologia Argumento, 29 (66), 383-399. 
MARIA DE FATIMA DE MATOS MAIA, NILTON SOARES FORMIGA, THATIANA MAIA TOLENTINO, CELINA APARECIDA GONÇALVES LIMA, BERENILDE VALÉRIA DE OLIVEIRA SOUSA

Van De Vijver, F., \& Leung, K. (1997). Methods and data analysis for cross-cultural research. Thousand Oaks, CA: Sage Publications. doi.org/10.1590/S0103-166X2008000100006

Vasconcelos, T. C., Gouveia, V. V., Pimentel, C. E, \& Pessoa, V. S. (2008). Condutas desviantes e traços de personalidade: testagem de um modelo causal. Revista Estudos de Psicologia, Campinas, 25(1), 55-65.

\section{Os Autores}

Maria de Fatima de Matos Maia é ddoutora, ddocente da Universidade Estadual de Montes Claros. Líder do Grupo Integrado de Pesquisa em Psicologia do Esporte, Exercício e Saúde, Saúde Ocupacional e Mídia. Montes Claros, Minas Gerais - Brasil. E-mail: fatima.maia204@ gmail.com. Correspondência: Campus Universitário Professor Darcy Ribeiro. Vila Mauricéia. 39.400-000 - Montes Claros/MG - Brasil. Tel: +55 (38) 3229-8000

Nilton Soares Formiga é doutor em Psicologia Social pela Universidade Federal da Paraíba. Atualmente é professor da Pós-graduação em Administração e Psicologia Organizacional (nível doutorado e mestrado) na Universidade Potiguar, Natal-RN, Brasil. 1. E-mail: nsformiga@yahoo.com.nsformiga@yahoo.com.

Thatiana Maia Tolentino é doutora - docente das Faculdades Santo Agostinho de Sete Lagoas. Membro do Grupo Integrado de Pesquisa em Psicologia do Esporte, Exercício e Saúde, Saúde Ocupacional e Mídia. Montes Claros, Minas Gerais - Brasil. E-mail: tatianam@hotmail.com.

Celina Aparecida Gonçalves Lima é mestre em Ciências da Saúde - Docente da Universidade Estadual de Montes Claros. Membro do Grupo Integrado de Pesquisa em Psicologia do Esporte, Exercício e Saúde, Saúde Ocupacional e Mídia. Montes Claros, Minas Gerais - Brasil. E-mail: celina.prof@bol.com.

Berenilde Valéria de Oliveira Sousa é mestre em Ciências da Saúde - Docente da Universidade Estadual de Montes Claros. Membro do Grupo Integrado de Pesquisa em Psicologia do Esporte, Exercício e Saúde, Saúde Ocupacional e Mídia. Montes Claros, Minas Gerais - Brasil. E-mail: berenilde.valeria7@ gmail.com.br

Recebido em: 29/04/2018

Aprovado em: 28/12/2018 